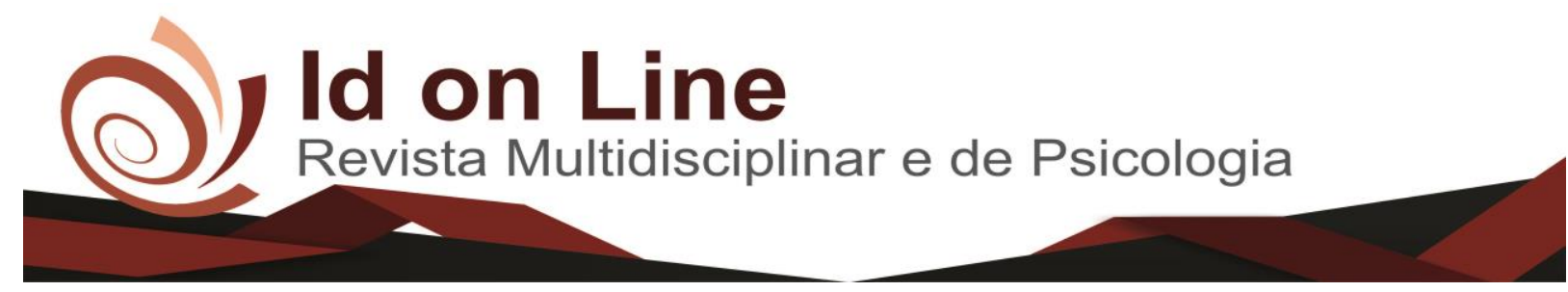

Artigo

\title{
Acidente de trabalho com exposição a material biológico em profissionais de Odontologia
}

\author{
Diogo Vieira de Sousa ${ }^{1}$; Marcelo Pereira da Rocha ${ }^{2}$
}

Resumo: Acidentes de trabalho com exposição a material biológico são previsíveis e podem ser evitados. Este trabalho teve como objetivo realizar uma revisão de literatura acerca dos acidentes de trabalho com exposição a material biológico entre profissionais dos serviços odontológicos, com foco voltado aos riscos, à notificação e medidas de prevenção. A pesquisa foi realizada em diversas bases de dados como Lilacs e Scielo. É elevada a subnotificação dos acidentes. Os riscos e as medidas de prevenção dos acidentes são conhecidos e precisam ser melhor divulgados. Os serviços de saúde devem adotar medidas de proteção da saúde de toda a equipe de trabalho, de modo a se proteger a saúde dos trabalhadores.

Palavras-chave: Exposição Ocupacional; Acidentes de Trabalho, Dentista

\section{Accident at work with exposure to biological material in dental professionals}

\begin{abstract}
Accidents at work with exposure to biological material are predictable and can be avoided. This work had as objective to conduct a review of the literature about the occupational accidents with biological material exposure among professionals of the dental services, with focus on risk notification and prevention measures. The survey was conducted in various databases like Lilacs and Scielo. Is high the underreporting of acidentes. The risks and the measures of prevention of accidents are well known and need to be better disseminated. Health services should adopt measures of protection of the health of the entire staff, so as to protect the health of workers.
\end{abstract}

Keywords: Occupational Exposure; Accidents at work, Dentist

\footnotetext{
${ }^{\mathbf{1}}$ Acadêmico do curso de Odontologia da Faculdade Independente do Nordeste (FAINOR) - Vitória da Conquista-Ba; Email: diogo_vs10@hotmail.com; Telefone para contato: (77) 99813-6905;

${ }^{2}$ Mestre em Saúde Coletiva (ISC/UFBA - Salvador, Ba); Especialista em Saúde Coletiva, em Planejamento e Práticas de ensino em Endodontia; Graduado em Odontologia e Biologia Sanitária (Univale). Sanitarista efetivo da Secretaria Estadual de Saúde da Bahia (SESAB); Docente e preceptor de cursos realizados pela SESAB; Membro efetivo da Comissão Intergestora Regional; Docente do curso de Odontologia da FAINOR; Email: cdbiomarcelo@yahoo.com.br; Telefone para contato: (77) 98809-5543
} 


\section{Introdução}

Acidente de trabalho com exposição a material biológico, potencialmente contaminado, é um sério risco aos profissionais da Odontologia e grave problema de saúde pública. São classificados como agravos à saúde do trabalhador, de notificação compulsória e exigem atendimento imediato ao acidentado (BRASIL, 2006).

Os trabalhadores de saúde ficam expostos a acidentes de trabalho devido aos procedimentos realizados nos serviços de saúde, com possíveis danos à integridade mental e física do trabalhador, inclusive doenças infecciosas. Profissionais como dentistas, técnicos e auxiliares de saúde bucal estão expostos a riscos de acidentes, principalmente causados por materiais perfurocortantes e fluidos biológicos (SILVA; MACHADO; SANTOS, 2011).

O Brasil possui uma política definida para a Saúde do Trabalhador (BRASIL, 2012). Objetiva a atenção integral à saúde do trabalhador, com ênfase na vigilância, visando a promoção e a proteção da saúde dos trabalhadores e a redução da morbimortalidade por agravos relacionados ao trabalho. Para o planejamento das ações, os agravos mais graves devem ser conhecidos. Por isso, orienta-se a notificação dos acidentes de trabalho, incluindose aqueles decorrentes de exposição a material biológico (BRASIL, 2014).

Ressaltam-se lacunas no conhecimento quanto à situação referente aos acidentes de trabalho com exposição a material biológico, especificamente entre profissionais dos serviços odontológicos. Este trabalho teve como objetivo realizar uma revisão de literatura acerca dos acidentes de trabalho com exposição a material biológico entre profissionais que atuam em serviços odontológicos, com foco voltado aos riscos, a notificação e medidas de prevenção.

\section{Material e Métodos}

Este trabalho foi conduzido por meio de revisão de literatura narrativa, utilizando-se publicações acerca de acidentes de trabalho com exposição à material biológico entre profissionais de odontologia. Foram consultados artigos e outros formatos de publicações, com acesso às bases de dados: Scielo, Bireme, Lilacs, Pubmed, Google Acadêmico e Periódicos CAPES. 
Foram selecionadas publicações nacionais e estrangeiras, de acesso gratuito, com texto completo, do período entre 2000 a 2016, nas línguas portuguesa e inglesa. Para a busca foram utilizadas as palavras-chave: odontologia, notificação de acidentes de trabalho e riscos ocupacionais, cadastradas nos Descritores em Ciências da Saúde (DeCS). Foram excluídos trabalhos com temática divergente dessa proposta, bem como os escritos em outros idiomas não citados acima, os que não possuíam texto completo, os que não tiveram acesso livre e as publicações anteriores a 2000.

\section{Revisão da Literatura}

O ambiente de trabalho saudável e a preservação das saúde dos trabalhadores são valiosos bens para as pessoas e os países. A saúde dos trabalhador contribui positivamente para a produtividade, motivação e satisfação no trabalho e, consequentemente, para a melhoria geral na qualidade de vida dos indivíduos e da sociedade (OMS, 2017).

\section{Riscos à Saúde dos Trabalhadores em Odontologia}

A prática da Odontologia abrange uma grande variedade de procedimentos, com diferentes níveis de complexidade, e expõe os profissionais a contato com secreções da cavidade oral, a exemplo de saliva, sangue, além de aerossóis, sendo fator de risco para a transmissão de infecções entre profissionais e pacientes (GARCIA; BLANK, 2008).

Os acidentes de trabalho com exposição a material biológico ocorrem quando o trabalhador entra em contato com materiais orgânicos, como sangue, entre outros, através das vias percutânea, mucosa e pele não íntegra. A partir desse contato, o trabalhador fica exposto a patógenos transmissíveis como do vírus da hepatite $\mathrm{B}(\mathrm{HBV})$, da hepatite $\mathrm{C}(\mathrm{HCV})$ e da imunodeficiência humana (HIV) (ANDRADE et al, 2013; ALVES-REZENDE; LORENZATO, 2000).

Os riscos de adoecimento variam de acordo com o tipo de exposição. Pode ocorrer por meio de lesões percutâneas, provocadas por instrumentos perfurantes e cortantes; acidentes com mucosas, quando há respingos envolvendo olho, nariz e boca; acidentes cutâneos, 
quando há contato do material biológico com pele não íntegra; e por mordeduras humanas, consideradas como exposição de risco quando envolvem a presença de sangue (BRASIL, 2014).

Estes acidentes podem ocorrer na rotina diária da equipe de saúde bucal, e frequentemente se devem à dinâmica dos procedimentos realizados na prática clínica, na qual envolve o manuseio de instrumentos perfurocortantes. Diversos fatores estão associados aos acidentes, como o estresse, pressa e nervosismo (GARCIA; BLANK, 2008).

$\mathrm{Na}$ Odontologia, os acidentes são frequentes tendo em vista que há uma constante manipulação em um campo restrito de visualização, a cavidade bucal, com objetos cortantes e pontiagudos, instrumentos rotatórios e ultrassônicos que elevam o risco de lesões e abrasões nas mãos e infecção por aerossóis contaminados e ainda, fragmentos e líquidos projetados, com risco de lesões oculares (ALVARES, et al, 2015).

As longas jornadas de trabalho e condições de trabalho dos cirurgiões-dentistas e auxiliares de saúde bucal fazem com que esses estejam expostos a uma vasta variedade de microrganismos que se encontram na saliva, sangue, vapores e aerossóis liberados pelos instrumentais rotatórios e até mesmo pelas vias aéreas dos pacientes (CHIODI; MARZIALE; ROBAZZI, 2007). Os riscos biológicos a que os trabalhadores da saúde bucal estão expostos podem causar infecções como, herpes, gripe, rubéola, sarampo, tuberculose, hepatites, sífilis, e a síndrome da Imunodeficiência Humana (BRASIL,2014).

A posição de trabalho do cirurgião-dentista e do auxiliar em saúde bucal e até mesmo do paciente podem contribuir para a ocorrência de acidentes. Esses podem acontecer quando equipamentos odontológicos não são utilizados com os devidos cuidados e até mesmo por movimentos inesperados do paciente. Os equipamentos de proteção individual como jaleco, luvas, óculos de proteção, máscara e gorro são importantes para prevenção de contaminações cruzadas (RIBEIRO; HAYASHIDA; MORIYA, 2007).

Uma exposição biológica a sangue e fluidos de origem ocupacional geram consequências, além do comprometimento físico a curto ou longo prazo. Podem trazer implicações de ordem emocional, social, familiar, profissional e até financeira. Diversas doenças podem surgir como consequência de acidentes levando a impactos na vida da vítima (ALVARES, et al, 2015).

Os acidentes com materiais biológicos são crescentes devido a diversas condições, como demasia da carga horária de trabalho, estresse relacionado ao estado emocional, tanto 
do profissional como do paciente atendido, e o desrespeito às normas de biossegurança (SILVA; MACHADO; SANTOS, 2011)

Cavalcante et al., (2013), identificaram os motivos para o acontecimento dos acidentes com exposição de material biológico: pouca experiência profissional, condições inadequadas de trabalho, ausência ou inadequação de equipamentos de proteção, cansaço físico e mental, necessidade de maior agilidade na realização das atividades de rotina, manuseio de material perfuro cortante, manipulação de material biológico, dentre outros.

Nascimento et al., (2012) também ressaltaram as principais causas que geram os acidentes de trabalho com exposição a material biológico com os profissionais da Odontologia, entre elas, a pressa, cansaço, nervosismo, repetição dos movimentos, excesso de trabalho e despreparo ou falta de atenção ao manusear equipamentos.

O principal material contaminante em acidentes com risco biológico em consultórios odontológicos é o sangue, seguido de vapores contidos no ar e da respiração, e fluidos dos próprios pacientes. $\mathrm{O}$ acidente de trabalho com risco biológico ocorre entre profissionais de diversas funções, não só na área da saúde, como também trabalhadores dos serviços de limpeza, estudantes e pesquisadores, sendo importante todos os casos serem registrados (GARBIN, et al, 2006). Entre os acidentes com risco biológico os causados por instrumentos perfurocortantes foram os mais frequentes (GARCIA; BLANK, 2006).

\section{Notificação dos Agravos Relacionados ao Trabalho}

$\mathrm{O}$ registro de acidentes constitui-se em importante instrumento de planejamento e reorientação das medidas de prevenção. A notificação criteriosa garante dados para pesquisas mais confiáveis e melhor conhecimento da situação pelo setor de vigilância à saúde, bem como pelo próprio serviço de saúde. Garante ao profissional acidentado o direito a receber um atendimento digno pós-exposição e possível acesso a benefícios trabalhistas (CHIODI; MARZIALE; ROBAZZI, 2007).

No Sistema de Informação de Agravos de Notificação (SINAN) devem ser notificados todos os casos, independente da forma de inserção do trabalhador no mercado de trabalho e de ser ou não segurado da Previdência Social. O SINAN possui a finalidade de recolher, propagar e dissipar os dados que foram produzidos nas três esferas do governo a fim de 
prover informações para análise do perfil da morbidade, contribuindo para o processamento de tomada de decisões (CAVALCANTE, et al.,2013).

O ingresso dos dados no SINAN é realizado por meio de ficha de notificação e ficha de investigação. Os formulários são padronizados e específicos para cada ocorrência (KOHN, et al, 2003). Além do registro no SINAN, deve-se emitir a Comunicação de Acidente de Trabalho (CAT), nos casos de trabalhadores vinculados à Previdência Social (SINAN, 2004).

A Portaria $\mathrm{n}^{\circ}$. 1.271 (BRASIL, 2014) definiu a lista nacional de notificação compulsória de doenças, agravos e eventos de saúde pública nos serviços de saúde públicos e privados. Os acidentes de trabalho com exposição a material biológico foram definidos como agravos de notificação compulsória pelos profissionais da saúde ou responsáveis pelos serviços de saúde públicos e privados que prestam assistência ao paciente, inclusive os de natureza odontológica (BRASIL, 2014).

Muitos acidentes de trabalho não são registrados, inclusive em ambientes odontológicos (MINISTÉRIO DA SAÚDE, 2006). O registro não ocorre devido a alguns fatores, como à falta de cooperação do próprio acidentado, medo de represálias por parte da empresa ou falhas da unidade de saúde onde o atendimento foi realizado, impossibilitando a percepção da real magnitude dos acidentes biológicos ocorridos e suas implicações na saúde do profissional (CCVAT, 2011).

Nas notificações do SINAN encontraram-se muitos dados ignorados e em branco. Reforça-se a necessidade da melhoria do sistema de investigação e registro de acidentes, visto que os registros e as inconsistências tornam o SINAN menos fidedigno na designação do perfil epidemiológico desses acidentes (GARCIA; BLANK, 2006).

Pesquisa conduzida por Machado-Carvalhais et al (2008) em relação à notificação, revelou que somente $16,7 \%$ dentistas efetivaram o registro por meio da Comunicação de Acidente de Trabalho (CAT), fato que resulta em baixo índice de ocorrência das estatísticas relativas a acidentes de trabalho, em especial, no campo da Odontologia. Bragança DPP, et al (2010), demonstraram a prevalência de acidentes de trabalho com exposição a material biológico em cirurgiões-dentistas (18,3\%) e auxiliares de saúde bucal, 12,8\%.

Salienta-se grande subnotificação dos agravos à saúde do trabalhador. Quando não é feito o registro do caso pós-exposição biológica, há prejuízos para o acidentado. Assim, perde-se o acompanhamento do trabalhador vitimado, seus possíveis direitos na previdência, dados sobre sua recuperação e o desfecho final do acidente (CAVALCANTE, et al., 2013). 


\section{Medidas de proteção da saúde dos trabalhadores}

A exposição a agentes biológicos representa grande risco para o cirurgião-dentista e sua equipe, por estarem em contato constante com fluídos e instrumentais perfurocortantes. Os serviços de saúde devem adotar medidas preventivas, como imunização e implantação de protocolos de biossegurança (OLIVEIRA; ALMEIDA, 2015).

O Ministério do Trabalho possui Norma Regulamentadora (NR) $n^{\circ} 32$, específica para serviços de saúde, com as diretrizes para a implementação de medidas de proteção e segurança à saúde dos trabalhadores e recomendações para identificação de riscos e adoção de medidas preventivas. Os serviços de saúde são responsáveis pela capacitação dos trabalhadores e acompanhamento da saúde dos mesmos (BRASIL, 2005).

Os acidentes de risco biológico ocorridos com profissional e pacientes devem ser tratados como casos de emergência/urgência médica. As condutas para quimioprofilaxia pelo vírus HIV e da hepatite B devem ser estabelecidas de imediato após a ocorrência do incidente, sempre que for necessário, a depender de avaliação do serviço de referência (AUSTREGÉSILO, et al, 2016).

Para que ocorra maior segurança no trabalho, todo profissional deve ter o cuidado no seu ambiente laborativo, com utilização de meios como os Equipamentos de Proteção Individuais (EPI) e Coletivos (EPC), disponibilizados pela empresa, manter a caderneta de vacinas sempre atualizada e realizar uma correta anamnese para constatar como está a saúde do paciente. Caso haja exposição a material biológico, iniciar condutas adequadas, o mais precocemente possível (MAGAGNINI; ROCHA; AYRES, 2011).

Ressalta-se ainda o papel das instituições de ensino10 em preparar profissionais de saúde mais capacitados para lidar com os riscos nos ambientes laborais, bem como em estabelecer regras e estratégias de intervenção a fim de se reduzir os riscos (PAIVA, 2017).

O Ministério da Saúde elaborou um protocolo (BRASIL, 2006) de condutas pósexposição a materiais biológicos no qual descreve os principais procedimentos a serem adotadas após o acidente, como os cuidados com a área exposta, avaliação do acidente (material orgânico, tipo de contato, conhecimento da fonte), orientações e aconselhamento ao acidentado (sorologia da fonte e do acidentado, situação do acidente) e notificação do acidente. 


\section{Discussão}

A equipe de saúde bucal é bastante vulnerável a acidentes de trabalho com exposição a material biológico potencialmente contaminado. É elevada a incidência desses acidentes, apesar da notificação ainda ser incipiente.

Trabalhadores que realizam procedimentos invasivos são mais vulneráveis a esse tipo de acidente (CAVALCANTE, et al., 2013). Riscos ocupacionais devem ser minimizados, visando à redução dos acidentes de trabalho. $\mathrm{O}$ reconhecimento dos riscos colabora para a implantação de medidas preventivas e programas para proteção da saúde do trabalhador (MEDEIROS JÚNIOR, 2005).

Os registros são relevantes instrumentos para a verificação, planejamento e melhorias para os acidentes de trabalho. A notificação aos órgãos de vigilância é importante para a produção de informações. Os dados coletados são importantes para redirecionar políticas públicas e implementação de medidas de prevenção, podendo-se contribuir com a diminuição das ocorrências (CHIODI; MARZIALE; ROBAZZI, 2007). Por isso, a notificação deve ser estimulada, pois contribui para melhor conhecimento da situação e adoção de políticas públicas de saúde (GARBIN, et al, 2006).

As investigações das circunstâncias dos acidentes de trabalho são um meio eficaz para diagnóstico e planejamento. Observações de aspectos relacionados aos acidentes, como horários e setores que mais ocorrem, profissionais mais expostos, a área do corpo mais atingida, são importantes para se compreender a realidade. É importante planejar e elaborar estratégias que possam prevenir a ocorrência de novos casos de acidentes, resultando em segurança para os trabalhadores da área em questão (OLIVEIRA; ALMEIDA, 2015).

Ainda não existe um conhecimento da extensão da gravidade dos acidentes de trabalho com exposição a material biológico em cirurgiões-dentistas e auxiliares em saúde bucal, tendo em vista a elevada subnotificação (CAVALCANTE, et al., 2013).

Sabe-se que os acidentes de trabalho com risco biológico são previsíveis e preveníveis (BRASIL, 2006). A prevenção dos acidentes de trabalho e procedimentos pós-exposição a material biológico são essenciais para a segurança do trabalhador (MAGAGNINI; ROCHA; AYRES, 2011). 
Deve-se realizar o encaminhamento dos trabalhadores acidentados aos serviços especializados. Ressalta-se a responsabilidade dos serviços de saúde em desenvolver programas de proteção da saúde dos trabalhadores.

\section{Considerações finais}

Por meio desta pesquisa foi possível conhecer aspectos relacionados a acidentes de trabalho com exposição a material biológico entre profissionais da área odontológica. A situação é preocupante tendo em vista a elevada subnotificação dos acidentes, fato que prejudica conhecer a real situação.

Os serviços de saúde devem adotar medidas de proteção da saúde de toda a equipe de trabalho, a fim de se evitar casos de acidentes com risco de contaminação por material biológico. A vigilância em saúde deve empreender esforços para acompanhar os serviços de saúde, estimulando a notificação dos agravos relacionados à saúde do trabalhador e exigindo o cumprimento das medidas de proteção da saúde dos trabalhadores.

\section{Referências}

OLIVEIRA, R. H. G.; ALMEIDA, T. F. DE A. Riscos Biológicos em Odontologia - uma revisão da literatura. Rev Bahiana Odonto. Salvador; v. 5, n. 1, 2015.

BRASIL. Ministério do Trabalho e Emprego. Portaria $\mathrm{n}^{\circ}$ 485, de 11 de novembro de 2005. Aprova a norma regulamentadora $n^{\mathbf{0}} 32$ (Segurança e saúde no trabalho em estabelecimentos de saúde) Disponível em: <http://www.mte.gov.br/legislacao/normas_regulamentadoras〉. Acesso em 09. Out.2017.

BRASIL. Ministério da Saúde. Secretaria de Atenção à Saúde. Brasília : Editora do Ministério da Saúde, 2006.

BRASIL. PORTARIA N ${ }^{\circ}$ 1.823, DE 23 DE AGOSTO DE 2012. Institui a Política Nacional de Saúde do Trabalhador e da Trabalhadora. Brasília. Ministério da Saúde, 2012. 
ORGANIZAÇÃO MUNDIAL DE SAÚDE. Saúde do Trabalhador: OMS, 2017. Disponível em: http://www.paho.org/bra/index.php?option=com_content\&view=article\&id=378:saudedo-trabalhador\&Itemid=595. Acesso em: 09. Out. 2017.

SEANI, N. DE P.; ZARONI, W. C. S.; LEITE, M. F.; BIANCHI, P. R.; PEREIRA, T. C. R. Acidentes ocupacionais com material biológico em Odontologia: uma responsabilidade no ensino. Revista da ABENO, v. 17, n. 3, p. 76-88, 2017.

ANDRADE, R. R. A.; ALMEIDA, R. A. C.; SAMPAIO, G. C.; PEREIRA, J. R. D.; ANDRADE, E.S.S. Ocorrência de acidentes com instrumentais pérfuro-cortantes em clínica odontológica na cidade do Recife-Pernambuco-Estudo-piloto. Rev. Cir. Traumatol. BucoMaxilo-Fac., v. 13, n. 2, p. 87-100, Camaragibe, 2013.

ALVES-REZENDE, M. C. R.; LORENZATO, F. Avaliação dos procedimentos de prevenção dos riscos biológicos por cirurgiões-dentistas. Rev Assoc Paul Cir Dent, v. 54, p. 446-54, 2000.

GARCIA, L. P.; BLANK, V. L. G. Condutas pós-exposição ocupacional a material biológico na odontologia. Rev Saúde Pública, v. 42, n. 2, p. 279-86, 2008. DOI: http://dx.doi.org/10.1590/S0034-89102008000200013

BRASIL. Portaria $n^{\circ} .1 .271$ de 06 de junho de 2014. Define a Lista Nacional de Notificação Compulsória de doenças, agravos e eventos de saúde pública nos serviços de saúde públicos e privados em todo território nacional, nos termos do anexo e dá outras providências. Diário Oficial da União, Seção 1, n. 108, p. 67-69, 09 jun. 2014.

ALVARES, J. K.; PINHEIRO, P. M. M.; SANTOS, A.F.; OLIVEIRA, G. L. Avaliação da completitude das notificações compulsórias relacionadas ao trabalho registradas por município polo industrial no Brasil, 2007 - 2011. Rev Bras Epidemiol, v. 18, n. 1, p. 123-36, 2015.

RIBEIRO, P. H. V.; HAYASHIDA, M.; MORIYA, T. M. Acidentes com material biológico entre estudantes de graduação em odontologia. Revista de odontologia da Universidade Cidade de São Paulo, v. 19, n. 3, p. 263-8, 2007.

GARCIA, L. P.; BLANK, V. L. G. Prevalência de exposições ocupacionais de cirurgiõesdentistas e auxiliares de consultório dentário a material biológico. Cad. Saúde Pública, v. 22, n. 1, p. 97-108, Rio de Janeiro, Jan, 2006

MAGAGNINI, M. A. M.; ROCHA, S. A.; AYRES, J. A. O significado do acidente de trabalho com material biológico para os profissionais de enfermagem. Rev Gaúcha Enferm. ,v. 32, n. 2, p. 302-8, 2011.

BRASIL. Portaria $n^{\circ} 777 / G M$ de 28/04/04. Dispõe sobre os procedimentos técnicos para a notificação compulsória de agravos à saúde do trabalhador em rede de serviços sentinela específica, no Sistema Único de Saúde - SUS. 
BRASIL. Ministério da Saúde. Secretaria de Atenção à Saúde. Departamento de Ações Programáticas Estratégicas. Notificação de Acidentes de Trabalho fatais, graves e com crianças e adolescentes. Brasília, 2006. Disponível em: http://bvms.saude.gov.br/bvs/publicacoes/protocolo_not_acidentes_trab.pdf

AUSTREGÉSILO, N. S.; CARVALHO, B. K. G.; MEDEIROS, A. R.; CARNEIRO, S. E. R.; SOUZA, G. C. A. Prevalência E Notificações De Acidentes De Trabalho Com Exposição A Material Biológico Na Odontologia. Revista Ciência Plural., v. 2, n. 1, p. 102-119, 2016.

CHIODI, M. B.; MARZIALE, M. H. P; ROBAZZI, M. L. C. C. Acidentes de trabalho com material biológico entre trabalhadores de unidades de saúde pública. Rev Latino-am Enfermagem., v. 15, n. 4, p. 123-129, 2007.

GARBIN, A. J. I.; PRESTA, A. A.; GARBIN, C. A. S.; LIMA, D.C. Ocurrencia de accidentes ocupacionales y conducta em la práctica odontológica. Revista Cubana de Salud y Trabajo., v. 7, n. 1-2, p. 29-33, 2006.

KOHN, W. G.; COLLINS, A. S.; CLEVELAND, J. L. HARTE, J. A.; EKLUND, K. J.; MALVITZ, D. M, et al. Guidelines for infection control in dental health-care settings - 2003. MMWR Recomm Rep., v. 52, p. 1-66, 2003.

SILVA, A. I. D.; MACHADO, J. M. H.; SANTOS, E. O. B.; et al. Acidente com material biológico relacionado ao trabalho: análise de uma abordagem institucional. Rev Bras Saúde Ocupac., v. 36, n. 124, p. 265-73, 2011. DOI: http://dx.doi.org/10.1590/S030376572011000200010

MINISTÉRIO DA SAÚDE (BR). Secretaria de Atenção à Saude. Exposição a material biológico. Brasília: Ministério da Saúde; 2006.

CENTRO COLABORADOR DE VIGILÂNCIA DOS ACIDENTES DE TRABALHO. Boletim epidemiológico acidentes de trabalho com exposição potencial a materiais biológicos. Informe do centro colaborador UFBA/ISC/PISAT - MS/DSAST/CGSAT. Edição n. 3, ano I, out. 2011.

CAVAlCANTE, C. A. A.; CAVAlCANTE, E. F. O.; MACÊDO, M. L. A. F.; CAVALCANTE, E. S.; MEDEIROS, S. M. Acidentes com material biológico em trabalhadores. Rev Rene., v. 14, n. 5, p. 971-9, 2013.

SISTEMA DE INFORMAÇÃO DE AGRAVOS DE NOTIFICAÇÃO. SINAN [acesso em 30 mar 2013]. Disponível em: http://dtr2004. saude.gov.br/sinanweb

GARCIA, L. P.; BLANK, V. L. G. Prevalência de exposições ocupacionais de cirurgiõesdentistas e auxiliares de consultório dentário a material biológico. Cad. Saúde Pública., v. 22, n. 1, p. 97-108, 2006. 
MEDEIROS JÚNIOR, A. Representação Social sobre o acidente de trabalho na área de saúde. [Tese]. Natal: Universidade Federal do Rio Grande do Norte: Programa de Pós Graduação em Ciências da Saúde; 2005.

BRAGANÇA, D. P. P.; FERNANDES, M. M.; SASSI, C.; FRANCESQUINI JÚNIOR, L.; DARUGE JÚNIOR, E. Condutas do cirurgião-dentista frente a acidentes biológicos. Odonto., v. 18, n. 35 , p. 37-44, 2010.

NASCIMENTO, L. S.; ASSUNÇÃO, L. R. S.; SILVA JÚNIOR, N. G.; PEDREIRA, E. N.; SILVA, R. L. C. Acidentes com perfuro cortantes na Faculdade de Odontologia da UFPA: visualização de um Cenário. Rev Odontol Bras Central., v. 21, n. 56, p. 463-467, 2012.

MACHADO-CARVALHAIS, H. P. M.; RAMOS-JORGE, M. L.; AUAD, S. M.; MARTINS, L. H. P. M.; PAIVA, S. M.; PORDEUS, I. A. Occupational exposure to potentially infectious biological material in a dental teaching environment. J Dent Educ., v. 72, n. 10, p. 12011208, 2008.

Como citar este artigo (Formato ABNT):

SOUSA, Diogo V de; ROCHA, Marcelo P. da. Acidente de trabalho com exposição a material biológico em profissionais de Odontologia. Id on Line Revista Multidisciplinar e de Psicologia, 2017, vol.11, n.38, p. 90-101. ISSN: 1981-1179.

Recebido: 06.10.2017

Aceito: 09.10.2017 